\title{
Nonequilibrium thermodynamics of erasure with superconducting flux logic
}

\author{
Olli-Pentti Saira $\odot,{ }^{1,2, *}$ Matthew H. Matheny, ${ }^{1}$ Raj Katti, ${ }^{1}$ Warren Fon, ${ }^{1}$ Gregory Wimsatt, ${ }^{3}$ James P. Crutchfield $\odot,{ }^{3}$ \\ Siyuan Han, ${ }^{4}$ and Michael L. Roukes ${ }^{1, \dagger}$ \\ ${ }^{1}$ Division of Physics, Mathematics, and Astronomy and Kavli Nanoscience Institute, \\ California Institute of Technology, Pasadena, California 91125, USA \\ ${ }^{2}$ Computational Science Initiative, Brookhaven National Laboratory, Upton, New York 11973, USA \\ ${ }^{3}$ Complexity Sciences Center and Department of Physics, University of California, Davis, One Shields Avenue, Davis, California 95616, USA \\ ${ }^{4}$ Department of Physics and Astronomy, University of Kansas, Lawrence, Kansas 66045, USA
}

(Received 30 September 2019; accepted 20 January 2020; published 3 March 2020)

\begin{abstract}
We implement a thermal-fluctuation-driven logical bit reset on a superconducting flux logic cell. We show that the logical state of the system can be continuously monitored with only a small perturbation to the thermally activated dynamics at $500 \mathrm{mK}$. We use the trajectory information to derive a single-shot estimate of the work performed on the system per logical cycle. We acquire a sample of $10^{5}$ erasure trajectories per protocol and show that the work histograms agree with both microscopic theory and global fluctuation theorems. The results demonstrate how to design and diagnose complex, high-speed, and thermodynamically efficient computing using superconducting technology.
\end{abstract}

DOI: 10.1103/PhysRevResearch.2.013249

Information storage and processing are vital in coordinating modern society. A considerable fraction (10\%) of the global electrical power output is spent on operating and cooling the required computing infrastructure [1]. On scales large and small, reduction and mitigation of the processor waste heat are critically important to high-performance computing. Two complementary strategies for developing an optimal computing platform [2] suggest themselves. The first improves the speed and energy efficiency of the hardware platforms through engineering advances and the second, a scientific endeavor, identifies and pursues the fundamental physical limits of computing machines. The latter originates most directly in the works of Landauer [3], who argued from a microscopic perspective that logically irreversible operations have an irreducible energy cost. This limit is approached, though, only when the clock rate of the computation is low enough to allow nearly adiabatic physical evolution [4-9]. Most generally, physically embedded computing requires a trade-off between efficiency and speed, among other factors [10].

A key advance in efficient nonadiabatic computing appeared with the fluctuation theorems (FTs) that exactly describe the thermodynamics of small systems, which are necessarily driven out of equilibrium by external controls during information processing [11,12]. Experimental tests of FTs have been performed in a variety of microscopic systems [13-19] naturally amendable to performing Landauer-

\footnotetext{
*Corresponding author: osaira@bnl.gov

†Corresponding author: roukes@ caltech.edu
}

Published by the American Physical Society under the terms of the Creative Commons Attribution 4.0 International license. Further distribution of this work must maintain attribution to the author(s) and the published article's title, journal citation, and DOI. efficient computation. However, a large discrepancy exists between the speed and complexity of the thermodynamically optimal systems, on one hand, and application-relevant but inefficient traditional processors, on the other. As a consequence, the experimental challenges of operating a Landauer-efficient processor so that its logical functionality and thermodynamic performance are measurable typically preclude complexity beyond one-bit logic. Here we perform a now-classic Landauer bit erasure experiment on a hardware platform that promises to obviate many such limitations: superconducting flux logic [20]. It is interesting to note that recent implementations of heat engines based on weakly anharmonic superconducting resonators [21,22] rely on altogether different operation principles compared to our device, which exhibits a strong nonlinearity due to flux quantization.

Exploiting the intrinsic advantages of superconducting flux circuits, our device not only allows for a faithful implementation of the idealized picture put forth by Landauer, but provides a number of practical and theoretical advantages. The magnetic fluxes threading the superconducting loops, though describing macroscopic phenomena, are true microscopic coordinates in the sense that other electronic degrees of freedom are frozen through condensation to a quantummechanical ground state. Static controls cause no dissipation on the device, as the magnetic fields are sourced with superconducting leads. The intrinsic clock speed of the system is set by the frequency of small oscillations around the potential minimum, i.e., the plasma frequency, which is of the order of $10^{10} \mathrm{~Hz}$. Industrial-scale fabrication en masse and coupling of a large number of flux logic cells is possible [23,24]. Owing to these features, high-performance processors implementing complex logical functions have been realized with superconducting architectures [25-27]. For studying the fundamental physics of computing, it is interesting to note that dynamics dominated by either classical or quantum effects can be 
(a)

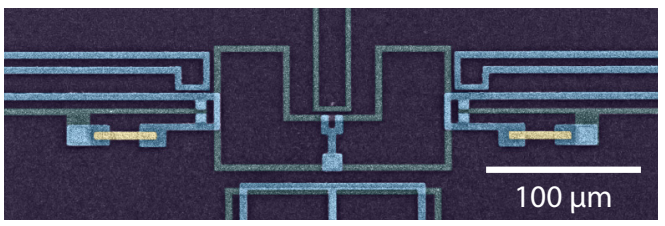

(b)

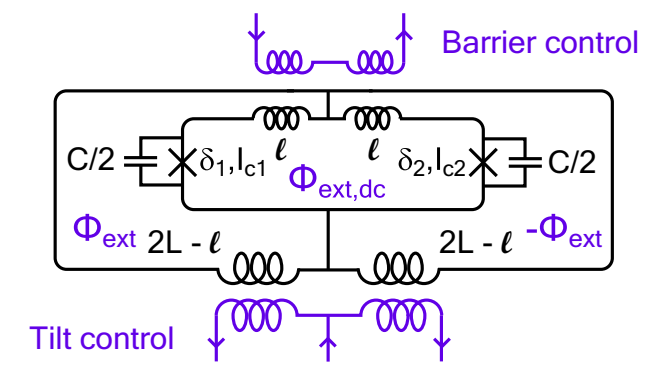

(c)

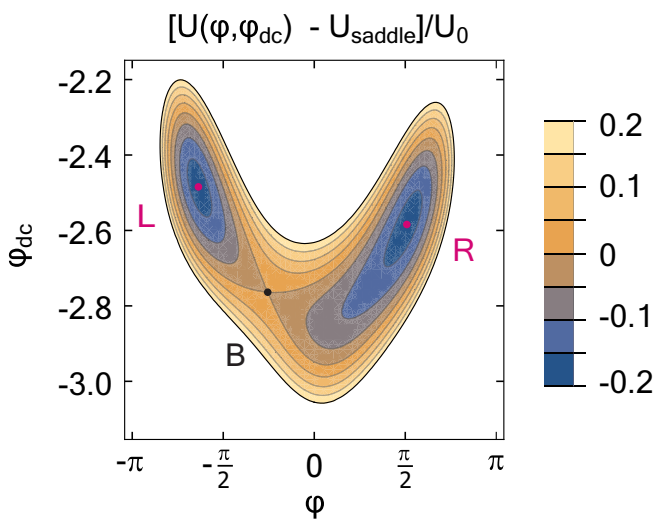

FIG. 1. Gradiometric flux logic cell. (a) False-color electron micrograph of the device, realized as a two-layer superconducting circuit on an insulating silicon substrate. (b) Simplified circuit schematic of the information-bearing subsystem. We take the dynamical coordinates to be the total magnetic fluxes $\varphi$ and $\varphi_{d c}$ threading the loops. (c) Contour plot of the potential calculated for the component values of the studied device and external bias fluxes $\left(\varphi_{x}, \varphi_{x, d c}\right)=(-0.1018,-2.5887)$ coinciding with the start of the bit erasure protocols studied later. Two local metastable minima ( $L$ and $R$, red dots) and the unique saddle point $(B$, black dot $)$ are marked.

accessed within this class of devices by a simple change of component values, external bias conditions, or temperature [28]. Finally, it is straightforward to engineer the dissipation acting on the remaining dynamical coordinates. Intrinsic dissipation in superconducting circuits has been found to be very low at frequencies up to $10 \mathrm{GHz}[29,30]$. Conversely, enhancing dissipation locally is straightforward through the inclusion of resistive normal metal shunts or coupling to external microwave ports.

In this work we study information erasure in a gradiometric flux logic cell [Fig. 1(a)]. The information-bearing subcircuit [Fig. 1(b)] is described by the standard two-dimensional flux qubit Hamiltonian [31-33]

$$
\begin{gathered}
H=\frac{Q^{2}}{2 C}+\frac{Q_{d c}^{2}}{C / 2}+U_{0} f\left(\varphi, \varphi_{d c}\right), \\
f\left(\varphi, \varphi_{d c}\right)=\frac{1}{2}\left(\varphi-\varphi_{x}\right)^{2}+\frac{\gamma}{2}\left(\varphi_{d c}-\varphi_{x, d c}\right)^{2} \\
+\beta_{L} \cos \frac{\varphi_{d c}}{2} \cos \varphi+\delta \beta \sin \frac{\varphi_{d c}}{2} \sin \varphi .
\end{gathered}
$$

The dynamical coordinates expressed in terms of the junction phases $\delta_{1}$ and $\delta_{2}$ are $\varphi=\left(\delta_{1}+\delta_{2}\right) / 2-\pi$ and $\varphi_{d c}=\delta_{2}-$ $\delta_{1}$. The bias terms are $\varphi_{x}=2 \pi \Phi_{\text {ext }} / \Phi_{0}-\pi$ and $\varphi_{x, d c}=$ $2 \pi \Phi_{e x t, d c} / \Phi_{0}$, where $\Phi_{0}$ is the magnetic flux quantum. Here $Q$ and $Q_{d c}$ are the common and differential-mode charges on the junction capacitors and conjugate to $\varphi$ and $\varphi_{d c}$, respectively. The potential parametrization is related to the circuit component values as follows: $U_{0}=\Phi_{0}^{2} / 4 \pi^{2} L, \gamma=$ $L / 2 l, \beta_{L}=2 \pi L\left(I_{c 1}+I_{c 2}\right) / \Phi_{0}$, and $\delta \beta=2 \pi L\left(I_{c 2}-I_{c 1}\right) / \Phi_{0}$, where $L$ and $2 l$ are the geometric inductances of the tilt and barrier pick-up loops, respectively, and $I_{c 1,2}$ are the critical currents of the two Josephson junctions.

With a suitable choice of the device parameters and the external bias point, the two-dimensional fluxoid potential has the required characteristics for implementing efficient bit storage and erasure. The theoretical potential calculated with calibrated device parameters [Fig. 1(c)] illustrates one of the basic requirements, namely, two metastable minima and the true two-dimensional nature of the system dynamics. In addition, the system allows for independent control of the tilt and the barrier height through the external control fluxes $\varphi_{x}$ and $\varphi_{x, d c}$. The barrier control has a large tuning range and allows the potential to be continuously deformed from two effectively isolated wells to a landscape with a single global minimum. We utilize this for device characterization.

Damping, and equilibrium noise associated with it, can be accounted for with a Langevin equation in the classical regime. However, in this work, the characterization experiments as well as the erasure trajectory data sets can be quantitatively explained by a simpler model that only involves the number and location of the critical points of the fluxoid potential. In the subsequent discussion, the labels $L, R$, and $B$ refer to the two local minima and the saddle point of the $f\left(\varphi, \varphi_{d c}\right)$ potential landscape, respectively. The right minimum is the one with the larger $\varphi$ coordinate. These points exist and are uniquely defined at all times during the erasure protocols, but not for all possible external controls. Furthermore, we define $U_{i}$ as the value of the potential term $U\left(\varphi, \varphi_{d c}\right)$ at the point $i$, and $U_{i j}=U_{j}-U_{i}$. Hence, $U_{L B(R B)}$ gives the barrier height for escape from the metastable minimum $L(R)$ and $U_{L R}$ is the energetic biasing of the double-well system.

The system under study is not overdamped, i.e., the $Q$ factor evaluated for oscillations in the metastable potential wells is not less than one. However, the energy relaxation time $Q / \omega_{0}=R C$, where $\omega_{0}$ is the plasma frequency and $R$ is the effective damping resistance of the logic cell, is much shorter than the timescale over which the external controls are changed, giving the system ample time to equilibrate during the execution of the protocols. This is equivalent to the validity of the Markovian activation-rate description of the interwell dynamics.

As the first step of the experimental device calibration, we exploit the periodicity of the flux response to set up an affine transformation between the idealized controls $\varphi_{x}$ and $\varphi_{x, d c}$ and the output voltages of waveform sources that drive the on-chip flux lines through an attenuator network. This transformation is applied implicitly throughout the experiments. For quantitative predictions, a straightforward minimum-tracking algorithm reproduces the global behavior of the trapped flux coordinate $\varphi$, including the characteristic $\left(\Phi_{0}, 2 \Phi_{0}\right)$ 


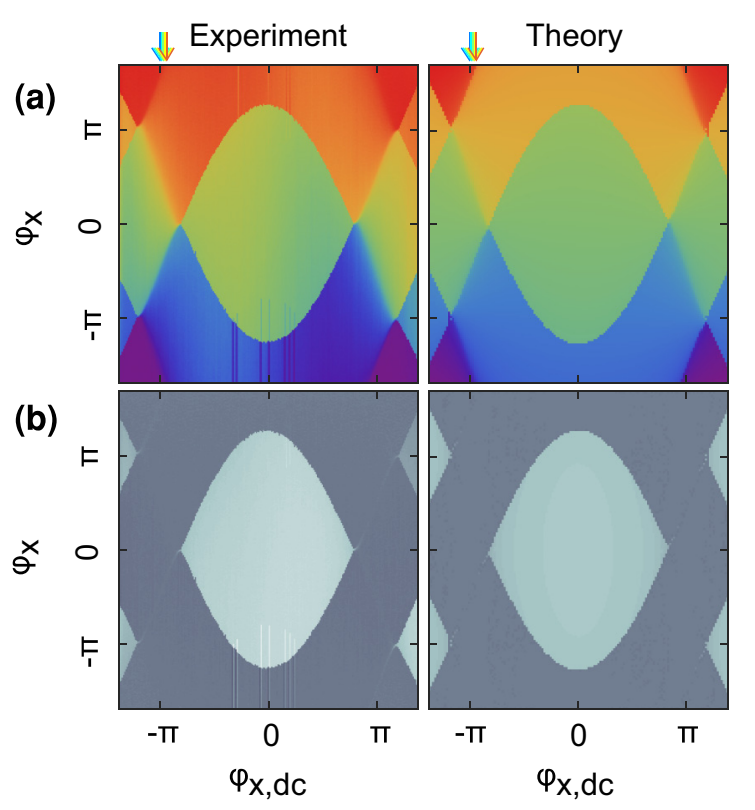

(c)

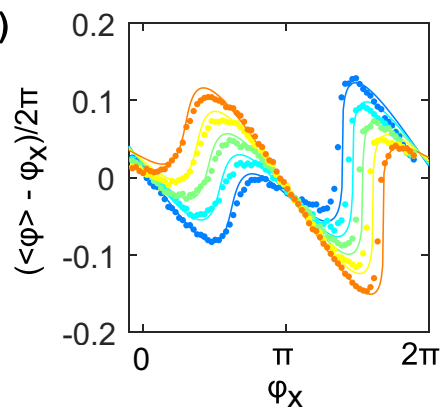

FIG. 2. Quasistatic response of the flux logic cell. (a) and (b) Two-dimensional scan of tilt and barrier controls $\left(\Phi_{x}\right.$ and $\Phi_{x, d c}$, respectively). Experimental plots (left) show the unprocessed phase of the local magnetometer readout. Theoretical plots (right), from Eqs. (1) and (2), show the coordinate $\varphi$ at a local minimum of the potential. A bidirectional sweep of tilt was performed for each value of the barrier control. Top panels (a) show the mean response to the two sweep directions. Bottom panels (b) show the response to the positive-direction sweep subtracted from the response to the negative-direction sweep, revealing metastability. (c) Detailed features of the flux response for a few different values of the barrier control [indicated by arrows on top of the (a) panels] in the nonhysteretic regime. Results of data are shown by markers and of theory by solid lines. The nonlinear flux-to-phase transfer function of the magnetometer has been inverted.

periodicity in control flux space [Fig. 2(a)], the number of local minima [Fig. 2(b)], and the nonlinear response of the $\varphi\left(\varphi_{x}\right)$ in the single-valued regime [Fig. 2(c)]. We determine the parameter values $\beta_{L}=6.2, \gamma=12$, and $\delta \beta=0.2$ entering Eq. (2) that yield the best agreement with experimental data. Here we utilize the fact that the mean response is insensitive to the value of $U_{0}$ and unaffected by moderate environmental noise when there is only one global minimum or when the barrier-crossing dynamics is frozen out.

In the case when two minima are separated by a moderate (several times $k_{\mathrm{B}} T$, where $k_{\mathrm{B}}$ is the Boltzmann constant) barrier, the thermally activated Markovian interwell transition rates are given by $[34,35]$

$$
\Gamma_{L, R}=\frac{\Omega}{2 \pi} \exp \left(-U_{L B, R B} / E_{\mathrm{esc}}\right),
$$

where $\Gamma_{L, R}$ is the escape rate from well $L(R), \Omega$ is the renormalized plasma frequency, and $E_{\text {esc }}$ is the escape energy scale. For thermally activated dynamics, $E_{\mathrm{esc}}=k_{\mathrm{B}} T$. A large body of theoretical results on the nature of information flow and architectural costs in physical computing devices has been derived for systems described by a time-inhomogeneous Markovian model [36,37]. Our superconducting device generates faithful realizations of such models in a system for which, moreover, the microscopic dynamics is understood in detail.

An important aspect of the technical implementation of the experiment is ensuring that the environmental fluctuations driving the barrier-hopping dynamics correspond to a true thermal bath. In particular, broad-spectrum electromagnetic backaction from the local DC superconducting quantum interference device (SQUID) magnetometer can cause nonthermal activation above the barrier. To characterize the device dynamics free from magnetometer backaction, we employ a time-domain pulse sequence [Fig. 3(a)] where we make a short excursion of duration $\tau$ to an extreme tilt configuration, thereby probabilistically causing the fluxoid particle to escape to the other minimum. We then return the tilt to the neutral setting $\left(U_{L R}=0\right)$ and determine the fluxoid state by a readout pulse. When performed under a sufficiently high barrier, ensuring that the readout pulse does not trigger state transitions at neutral tilt, the escape rate during the maximum tilt can be determined from the observed transition probability $p$ as

$$
\Gamma=-\frac{\ln (1-p)}{\tau} .
$$

Adherence to the activation rate model [Eq. (3)] can be verified by determining the escape rate as a function of barrier heights (evaluated from the tilt amplitude and polarity and system parameters) at different temperatures. We performed the experiment at a constant barrier control $\varphi_{x, d c} / 2 \pi=-0.3778$ at temperatures up to $600 \mathrm{mK}$. The data display the expected exponential dependence of the escape rate on the barrier height [Fig. 3(b)]. Due to strong asymmetry in the potential, measuring the escape rate in both directions (from the left minimum to the right and vice versa) serves as an additional check of the validity of the extracted model parameters. Next we fit the escape energy at each temperature and polarity independently. We find the escape energy to be proportional to the sample temperature above $200 \mathrm{mK}$ [Fig. 3(c)]. We take this proportionality to be proof of thermally activated dynamics. The common prefactor $U_{0}$ of the potential can be determined with a zero-intercept fit of the dimensionless escape energy in the proportional regime. We obtain $U_{0} / k_{\mathrm{B}}=56.3 \mathrm{~K}$ (positive polarity) and $U_{0} / k_{\mathrm{B}}=$ $56.7 \mathrm{~K}$ (negative polarity), corresponding to $L \approx 140 \mathrm{pH}$. The low-temperature saturation corresponds to temperatures $T_{c r}=103 \pm 2 \mathrm{mK}$ (positive polarity) and $T_{c r}=105 \pm 2 \mathrm{mK}$ (negative polarity).

A fundamental explanation of the low-temperature saturation is the transition from thermally activated dynamics to macroscopic quantum tunneling (MQT). Within this 

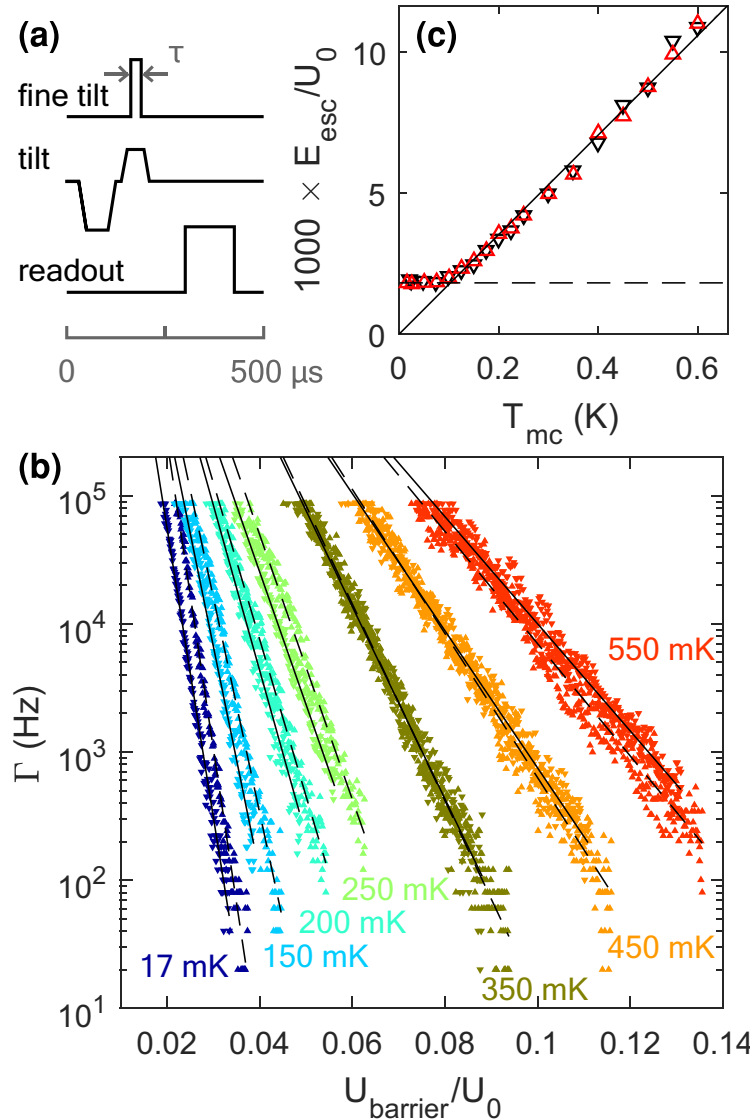

FIG. 3. Backaction-free escape dynamics. (a) Pulse sequence used for determining the escape rates. The sequence begins with a deterministic reset. The fine tilt pulse is superimposed with the tilt waveform with a large attenuation. (b) Escape rate as a function of the theoretical barrier height during the fine tilt pulse at different sample temperatures (markers). The experiment was performed at barrier setting $\varphi_{x, d c} / 2 \pi=-0.3778$ using both positive and negative polarity tilt pulses (upward and downward triangles, respectively). Lines are fits to data (solid and dashed for + and - polarity, respectively). (c) Escape energy, extracted as the inverse negative slope of the escape rate data, as a function of temperature, separately for + and - polarity data (upward and downward triangles, respectively). The solid line is a zero-intercept fit to data at $T>250 \mathrm{mK}$ and the dashed horizontal line indicates the low-temperature saturation level.

interpretation, $T_{c r}=\hbar \omega_{0} / 2 \pi k_{\mathrm{B}}$ [38-40], and the plasma frequency $\omega_{0} / 2 \pi$ of the system is approximately $13.6 \pm 0.4 \mathrm{GHz}$ at the operation points used for the escape rate experiments. Alternatively, we can estimate the capacitance $C$ from the total junction area $\left(11.8 \mu \mathrm{m}^{2}\right.$, based on a high-magnification scanning electron micrograph) and the nominal specific capacitance $45 \mathrm{fF} / \mu \mathrm{m}^{2}$ of the junction fabrication process. This yields $C=530 \mathrm{fF}$ and $\omega_{L C} / 2 \pi=$ $(2 \pi \sqrt{L C})^{-1}=18.5 \mathrm{GHz}$. In general, the plasma frequency $\omega_{0}$ differs from $\omega_{L C}$ due to varying local curvature of the potential [Eq. (1)]. The experimental $\omega_{0} / \omega_{L C}$ ratio is $0.74 \pm 0.02$, which is close to the theoretical values ranging between 0.63 and 0.73 for the different tilt amounts and polarities used in the experiment. We use the $\omega_{0}$ value given by the MQT experiment for the remainder of our analysis. Macroscopic resonant tunneling [41] peaks were not resolvable, presumably due to

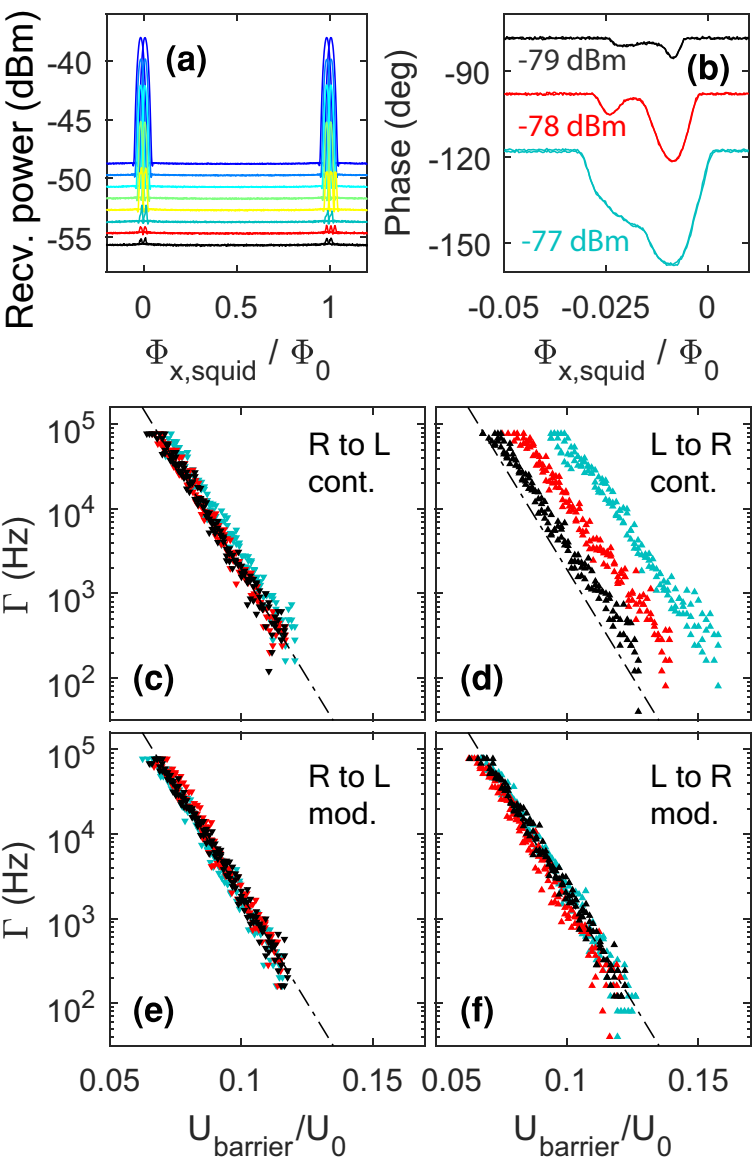

FIG. 4. Magnetometer readout with different probe powers and backaction on system dynamics at $T=500 \mathrm{mK}$. (a) Flux modulation curves for -79 to $-72 \mathrm{dBm}$ incident power, in 1-dBm steps. Pickup from magnetometer bias to tilt and barrier fluxes has not been compensated. (b) Phase response for the three lowest powers. Data have been offset by $20^{\circ}$ per $\mathrm{dBm}$ for clarity. (c) and (d) Escape rates at $\varphi_{x, d c} / 2 \pi=-0.3778$ under continuous readout for the three lowest powers. (e) and (f) Backaction-free pulsed readout under otherwise identical conditions. The operation point has been chosen so that the logical state $R$ is at the peak of the modulation curve. Consequently, the magnetometer is (close to) a zero-voltage state in the logical state $L$. The dash-dotted line is the same in all panels and serves as a guide for the eye.

small level separation in terms of tilt flux $\left(4.7 \times 10^{-4} \Phi_{0}\right)$. All further experiments are performed at a temperature of $500 \mathrm{mK}$, firmly in the thermal activation regime. At this temperature, the thermal energy is $k_{\mathrm{B}} T=6.9 \times 10^{-24} \mathrm{~J}$.

To extract work statistics for bit erasure at the singletrajectory level, the system state must be continuously tracked throughout the protocol. Therefore, we characterize the logical state-dependent backaction of the local magnetometer when read out with a continuous low-power sinusoidal $(f=$ $10 \mathrm{MHz}$ ) waveform. The probing frequency was chosen so that it is in the passband of an AC-coupled cryogenic SiGe preamplifier. The preamplifier output was demodulated with a rf lock-in-amplifier into two zero-i.f. quadrature channels that were subsequently digitized. The magnetometer SQUID was not part of a resonant circuit. Hence, the observed flux modulation characteristics [Figs. 4(a) and 4(b)] as well the 

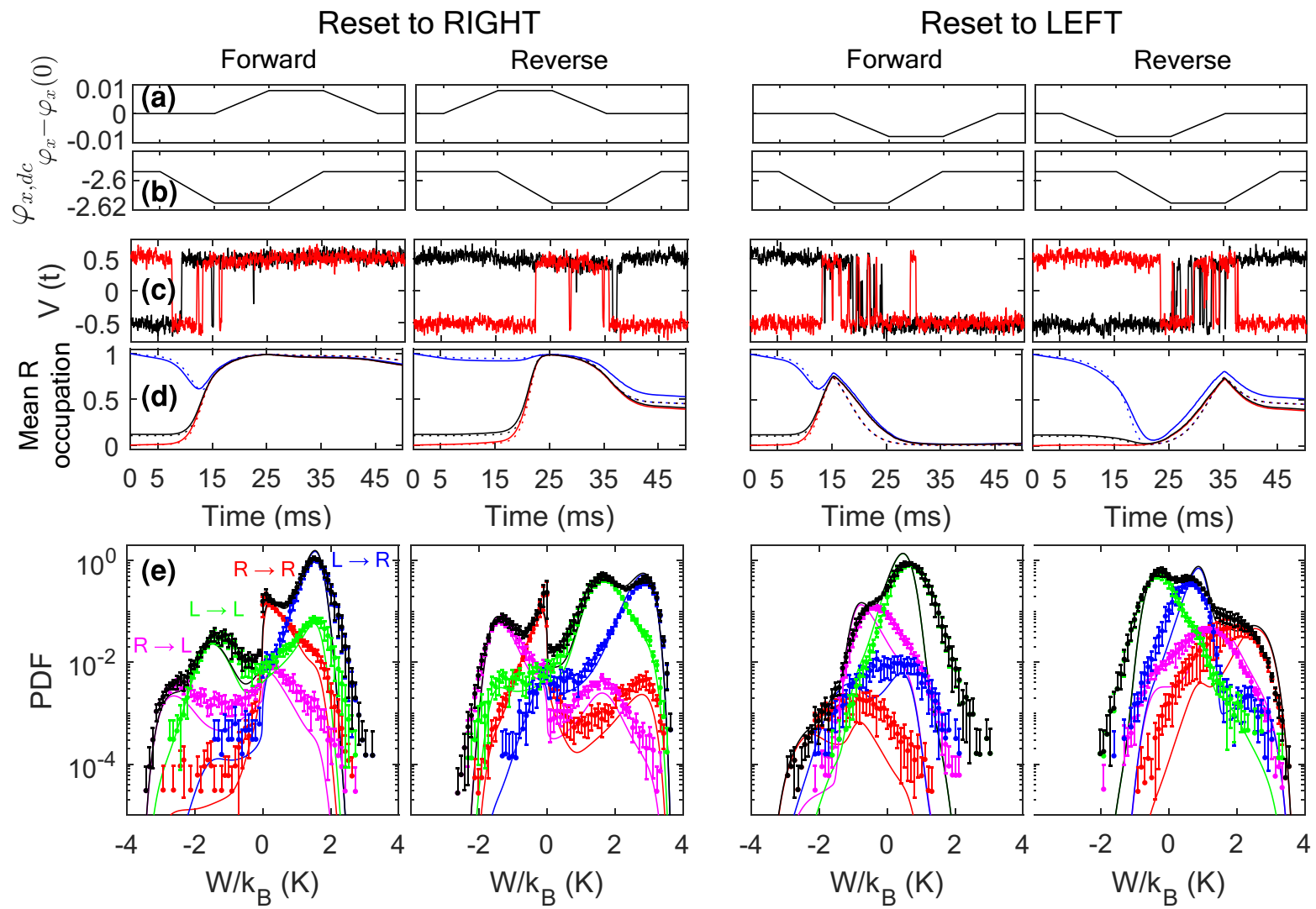

FIG. 5. Bit erasure at $T=500 \mathrm{mK}$. (a) and (b) Time-domain waveforms applied to tilt and barrier controls. The reverse protocol is obtained by reversing both control channels in time. The reset protocol targeting state $L$ has the polarity of the tilt waveform inverted. (c) Two randomly chosen magnetometer traces. (d) Average occupation of the state $R$ throughout the protocol when the initial state is $R$ (blue), $L$ (red), or an equilibrium mixture of the idling potential (black). The average is calculated from $10^{5}$ experimental trajectories (solid lines) and theory (dashed lines). (e) Distribution of work $W$ from $10^{5}$ experimental trajectories (markers) and from Markovian theory (lines). Full distribution (black) and conditional distributions based on initial and final states (magenta, green, red, and blue for trajectories of $R \rightarrow L, L \rightarrow L, R \rightarrow R$, and $L \rightarrow R$ type, respectively) are shown. Here PDF denotes probability density function.

nature of its backaction on the flux logic cell resemble those of a current-biased DC SQUID. From similar data, we extract a magnetometer flux shift due to the logical transition at $\varphi_{x, d c}=$ 0 to be $0.024 \Phi_{0}$, and the mutual inductance between the magnetometer and the flux logic cell $M=0.0254 L \approx 3.6 \mathrm{pH}$. From a separate low-temperature DC four-wire characterization of the readout SQUID, we determine its shunt resistance $R_{\text {shunt }}=2.1 \Omega$. We estimate the $Q$ factor due to shunt-induced damping of the flux dynamics as [42]

$$
Q_{\text {shunt }}=\frac{R_{\text {shunt }} L}{\omega_{0} M^{2}} \approx 260,
$$

where we have used the approximation $\omega_{0} \approx \omega_{L C}$, thus neglecting the contribution from the Josephson inductance. Noting that the chip contains two nominally identical readout circuits placed symmetrically with respect to the logic cell, we obtain an upper bound on the intra-well relaxation timescale $Q / \omega_{0} \leqslant Q_{\text {shunt }} / 2 \omega_{0}=9.5 \mathrm{~ns}$, where the total $Q$ includes all damping mechanisms. This sets the fundamental limit on what constitutes an adiabatic evolution in the system. However, the reset protocols studied in this paper are many orders of magnitude slower, ensuring that the timing of the logical state transitions can be accurately determined from the finitebandwidth magnetometer output.
The potentially harmful nonequilibrium backaction from a DC SQUID appears in the form of wideband microwave radiation with a complex spectrum peaked at $\omega_{J}=2 \mathrm{eV} / \hbar$ and harmonics, where $V$ is the DC voltage developed over the junction. For low-amplitude probing currents, it is possible to choose the magnetometer flux bias in such a way that the SQUID is in a finite-voltage state for logical state $L$ and in the zero-voltage state for logical state $R$. This configuration would be expected to result in logical-state-dependent backaction. We quantify the backaction by repeating the earlier escaperate experiment with both continuous and pulse-modulated readout at $T=500 \mathrm{mK}$. The data [Figs. 4(c)-4(f)] are in agreement with the model of readout backaction laid out above. We find only the $L$-to- $R$ escape under continuous readout [Fig. 4(d)] to be affected. The escape rate appears to be enhanced by a constant power-dependent factor, but the effective temperature, quantified by the slope of the $\Gamma$ vs $U_{\text {barrier }}$ characteristic, is not affected. Guided by this characterization, we choose an incident readout power of $-79 \mathrm{dBm}$ (to $50 \Omega$ load) for the continuous monitoring of stochastic bit erasure trajectories. At this power level, the rate enhancement is equivalent to a sub- $k_{\mathrm{B}} T$ change in the energetics of the system, while the signal-to-noise ratio remains sufficient for fast discrimination of the logic state [Fig. 5(c)]. 


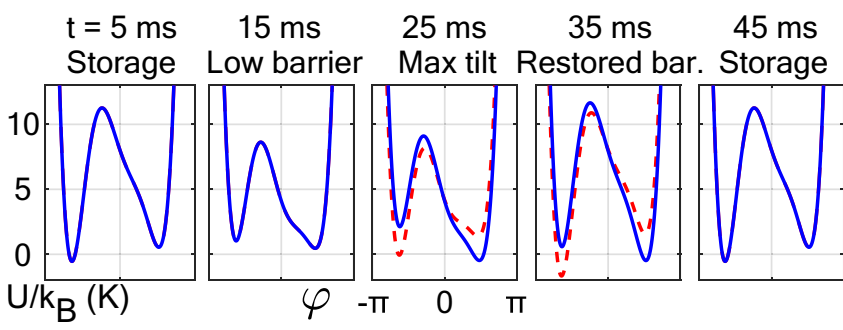

FIG. 6. One-dimensional projection of the potential at key stages of the reset-to- $R$ (blue solid line) and reset-to- $L$ (red dashed line) protocols. The plotted quantity is the potential as a function of the $\varphi$ coordinate evaluated along a curve in $\left(\varphi, \varphi_{d c}\right)$ space that passes through the left minima, the saddle point, and the right minima (in this order) and is parallel to the local principal axis of curvature at these points. A constant offset of $71.4 \mathrm{~K}$ has been subtracted for clarity.

To study the work statistics of bit erasure, we implement the reset protocol used in Ref. [5]. The protocol starts from and ends in a storage state. Logical state reset is realized by piecewise linear controls applied to the tilt and barrier channels. Efficient and fast reset is achieved by changing the controls in a particular sequence: drop barrier-tilt-raise barrier-untilt [Figs. 5(a) and 5(b) show the waveforms; Fig. 6 shows the induced potentials]. With the control waveform shapes fixed, one still has a choice of their duration, flux offset, amplitude, polarity, and directionality.

We derive three transformed versions of the basic protocol that implements reset-to- $R$ functionality: reset-to- $L$, obtained by inverting the polarity of the tilt waveform while maintaining the same offset $\varphi_{x}^{L}(t)=\varphi_{x}(0)-\left[\varphi_{x}^{R}(t)-\varphi_{x}(0)\right]$. In addition, for both polarities, the reversed protocol is obtained by time reversing both the tilt and barrier waveforms. Importantly, due to the finite- $\delta \beta$ term in the Hamiltonian [Eq. (1)], reversing the signs of the tilt control $\varphi_{x}$ and the longitudinal coordinate $\varphi$ does not result in an equivalent potential landscape. Hence, the reset-to- $L$ and reset-to- $R$ protocols give rise to a different distribution of microscopic trajectories. Snapshots of the potential at key stages of the reset protocols are shown in Fig. 6.

In the infinite-time limit, an application of this protocol with appropriate scaling of the controls results in a Landauerefficient reset with Gaussian work statistics. For a finiteduration protocol with ideal control of the energetics, the resulting work histogram is bimodal and displays characteristic features that can be traced back to different substages of the protocol [43]. In our experiment, the asymmetry of the Hamiltonian leads to a nontrivial functional dependence of the energetics (in particular, $U_{L R}$ ) on the external controls $\left(\varphi_{x}, \varphi_{x, d c}\right)$, giving rise to complex multimodal work distributions [Fig. 5(e)].

The important timescales of the experiment were chosen to satisfy

$$
\tau_{\text {readout }} \ll \Gamma_{\text {tilt }}^{-1} \ll \tau_{\text {total }},
$$

where $\tau_{\text {readout }} \approx 0.1 \mathrm{~ms}$ is the time needed to detect a logical transition, $\Gamma_{\text {tilt }}$ is the typical transition rate during the lowbarrier tilting phase (15-25 $\mathrm{ms}$ in protocol time), and $\tau_{\text {total }}$ is the total duration of the protocol. The first condition ensures that the magnetometer can track the system dynamics and the second ensures that the system can sample both wells during the protocol execution. For the protocols studied here, $\Gamma_{\text {tilt }} \approx 1 \mathrm{kHz}$. Finally, we choose $\tau_{\text {total }}=50 \mathrm{~ms}$. This allows us to collect $N=10^{5}$ trajectories for all four protocol transformations with a total measurement time of $12 \mathrm{~h}$ with $50 \%$ duty cycle. For the duration of the acquisition, the system is run with open-loop control.

We monitor the magnetometer output continuously during the execution of each reset protocol. Two randomly chosen traces are shown in Fig. 5(c). The polarity of the magnetometer response is such that the left logical state corresponds to a positive output voltage. We classify the instantaneous logical state according to the sign of the magnetometer signal. Evaluating the mean occupation of either logical state (here we choose $R$ ) as a function of time results in, over the $10^{5}$ experimental trajectories, a smoothly varying curve [Fig. 5(d)].

Evidently, logical state transitions correspond to the zero crossings in the magnetometer signal. We will use this symbolic representation of the system state to derive a pertrajectory work estimate. Consider a trajectory that starts in state $s_{0} \in\{L, R\}$ and involves $n$ state transitions such that the $i$ th transition occurs at time $\tau_{i}$ and takes the system to state $s_{i} \in\{L, R\}$. Defining $\tau_{0}=0$ and $\tau_{n+1}=\tau_{\text {total }}$, we write the per-trajectory work as

$$
\begin{aligned}
W & =\sum_{i=0}^{n}\left[U_{s_{i}}\left(\tau_{i+1}\right)-U_{s_{i}}\left(\tau_{i}\right)\right] \\
& =\left[U_{s_{n}}(0)-U_{s_{0}}(0)\right]-\sum_{i=1}^{n}\left[U_{s_{i}}\left(\tau_{i}\right)-U_{s_{i-1}}\left(\tau_{i}\right)\right] .
\end{aligned}
$$

The second equality makes use of the fact that the potentials at $t=0$ and $t=\tau_{\text {total }}$ are identical. The latter form illustrates that $W$ can be expressed as a sum of $U_{L R}\left(\tau_{i}\right)$ terms with alternating signs.

Even though our experimental flux traces consist of discrete fluxoid state transitions, the underlying dynamical coordinates are continuous. Evaluation of Eq. (8) gives an accurate estimate of the true microscopic work, provided (i) two metastable minima exist throughout the protocol, (ii) the system has time to locally equilibrate between logical transitions, (iii) the changes in control parameters are slow compared to the internal equilibration time, and (iv) the potential landscape is only weakly perturbed. The details of this argument in the context of superconducting flux logic are laid out in Ref. [43]. An equivalent approach is commonly used in studies of nonequilibrium thermodynamics in single-electron devices $[19,44]$. Numerical Langevin simulations of a doublewell system satisfying the above conditions confirm that the work distribution evaluated with the discretized formula in Eq. (8) agrees with that obtained for the microscopic work evaluated with continuous coordinates.

For the parameters of this experiment, the experimental initial tilt offset and the asymmetry of the potential give rise to a nonzero $U_{L R}(0) / k_{\mathrm{B}}=1.01 \mathrm{~K}$. We determine this initial energy offset based on the equilibration of the left and right state populations during the first tenth of the protocol, for which the system is in the storage state. Conversely, weighting 
the left and right initial conditions according to the Boltzmann factor for this energy offset results in a steady occupation in the initial idling period [Fig. 5(d), black line]. We use the same Boltzmann-factor weighing when aggregating the work histograms. With this weighting, fluctuation theorems are satisfied by the quantity $W$ defined above. Note that the first term of Eq. (8) vanishes if the potential in the initial storage state is degenerate, i.e., if $U_{L R}(0)=0$.

The per-trajectory work estimate is based on the calibrated potential, but does not require a model of the system dynamics. Given that we in addition calibrated the two-state activation rate model, we can use that knowledge to predict the system's time-domain response to the erasure protocols. The renormalization of the rates due to damping and local curvature of the potential $[31,34,45]$ is a much smaller effect than the variance due to uncertainty in the model parameters. Consequently, we substitute $\Omega=\omega_{0}$ as the prefactor in Eq. (3). Such predictions are included in the mean occupation plots of Fig. 5(d) as dashed lines and the work histograms of Fig. 5(e) as solid lines. This simple dynamical model reproduces with good accuracy the mean occupations throughout the protocols as well as the locations and relative weights of the peaks in the multimodal work histograms. One can easily discern, however, that the agreement is worse for the reset-to- $L$ family of protocols. The reason for the disagreement is not clear at the moment, but similar features can be observed in other data sets acquired from the same device.

To quantify the effect of slow flux offset drifts, we process the data in ten chunks of $10^{4}$ consecutive trajectories and plot the mean and $2 \sigma$ confidence intervals for each bin of the work histograms [Fig. 5(e)]. Left and right initial conditions have been weighted according to the Boltzmann factor defined above. We include the per-bin uncertainties in the evaluation of the Crooks-relation [12] ratios $\rho=P_{f w d}(W) / P_{\text {rev }}(-W)$ using standard error propagation formulas (Fig. 7). When evaluated in this manner, the confidence intervals also include the statistical uncertainty due to finite sampling, dominating the uncertainty for low-count bins. The fact that the Crooksrelation ratios fall on the expected line $\ln (\rho)=k_{\mathrm{B}} T$ within the error bars is another indication that our model of the microscopic energetics of the flux logic system is correct.

We characterize the thermodynamic efficiency of the protocols by comparing their mean work expenditure $\langle W\rangle$ to the change in free energy. Without loss of generality, we write the Helmholtz potential of the two-state system as

$$
\begin{aligned}
A & =U-T S \\
& =p_{R} U_{L R}+k_{\mathrm{B}} T\left[p_{R} \ln p_{R}+\left(1-p_{R}\right) \ln \left(1-p_{R}\right)\right],
\end{aligned}
$$

where $U_{L R}$ is the energetic bias defined earlier and $p_{R}$ is the occupation probability of the $R$ state. For the asymmetric potential prevailing at the end points of the protocols, the equilibrium $R$ state occupation is $p_{\mathrm{eq}}=\{1+$ $\left.\exp \left[U_{L R}(0) / k_{\mathrm{B}} T\right]\right\}^{-1}=0.117$ and the equilibrium entropy is $S_{\text {eq }}=0.360 k_{\mathrm{B}}$. The minimum work expenditure of an erasure protocol that operates on a thermal ensemble of input bits and targets either the $R$ or $L$ state is given by the free-energy difference $\Delta A_{R}=A(1)-A\left(p_{\mathrm{eq}}\right)=2.15 k_{\mathrm{B}} T$ or $\Delta A_{L}=A(0)-$ $A\left(p_{\text {eq }}\right)=0.124 k_{\mathrm{B}} T$, respectively, where $A(p)$ denotes the value of $A$ for $p_{R}=p$. Conversely, the corresponding reverse,

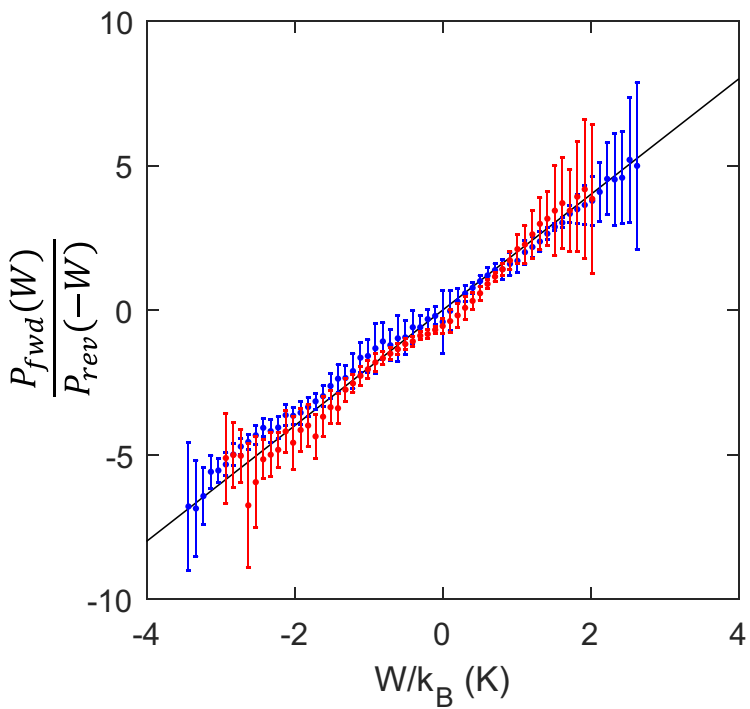

FIG. 7. Crooks relation ratio of the probability densities of pertrajectory work in the forward and reverse directions (see the text for definitions). The experimental ratio is for bit reset targeting the $R$ and $L$ states (blue and red markers, respectively) and the fluctuation theory prediction is $\exp \left(W / k_{\mathrm{B}} T\right)$ with $T=500 \mathrm{mK}$ (solid line).

i.e., bit creation, protocol can extract a maximum amount of $\Delta A_{R(L)}$ of work per bit from a pure ensemble of the correct input state. For an experimental or simulated protocol, we define $\Delta A=A\left(p_{\text {final }}\right)-A\left(p_{\text {init }}\right)$, where the final occupation $p_{\text {final }}$ is calculated for an assumed initial occupation $p_{\text {init }}$ from the observed transition probabilities $P\left[s\left(\tau_{\text {total }}\right)=i \mid s(0)=j\right]$, where $i, j \in\{L, R\}$. Similarly, we calculate $\langle W\rangle$ using in addition the observed conditional expected works $E[W \mid s(0)=$ $\left.j \wedge s\left(\tau_{\text {total }}\right)=i\right]$. The figures of merit for individual protocols are given in Table I. Finally, to aggregate the numbers into an information-theoretic fidelity, we consider a mixture of the

TABLE I. Work expenditures and free-energy differences for the bit erasure and creation protocols. All energies are given in units of $k_{\mathrm{B}} T$ at $T=500 \mathrm{mK}$. Experimental values are derived from the experimental trajectory data, simulated values are given by the Markovian two-state model, and ideal values are the theoretical optimum for an ideal implementation of the protocol. For bit erasure protocols, the input ensemble is assumed to be a thermal distribution. For bit creation protocols, the input is assumed to be a pure ensemble of the indicated state.

\begin{tabular}{lccccccc}
\hline \hline & $\langle W\rangle$ & $\Delta A$ & $\langle W\rangle / \Delta A$ & & $\langle W\rangle$ & $\Delta A$ & $\langle W\rangle / \Delta A$ \\
\cline { 2 - 4 } \cline { 6 - 7 } Protocol & \multicolumn{3}{c}{ Right } & & & \multicolumn{3}{c}{ Left } \\
\hline Erase & & & & & & \\
Expt. & 2.50 & 1.52 & 1.65 & & 1.08 & 0.06 & 17.1 \\
Sim. & 2.53 & 1.74 & 1.45 & & 0.55 & 0.09 & 6.20 \\
Ideal & 2.15 & 2.15 & 1 & & 0.12 & 0.12 & 1 \\
Create & & & & & & \\
Expt. & -0.93 & -1.64 & 0.57 & & 0.17 & 0.11 & \\
Sim. & -1.39 & -1.79 & 0.77 & & 0.52 & 0.23 & \\
Ideal & -2.15 & -2.15 & 1 & -0.12 & -0.12 & 1 \\
\hline \hline
\end{tabular}


right and left processes with respective weights of $p_{\text {eq }}$ and $1-p_{\text {eq }}$. With this weighting, the free-energy change for ideal bit erasure (creation) is purely entropic and equals $\pm k_{\mathrm{B}} T S_{\text {eq }}$. The aggregate experimental erasure process achieves $\Delta A=$ $0.233 k_{\mathrm{B}} T$ and $\langle W\rangle=1.25 k_{\mathrm{B}} T$ and consequently operates at 5.4 times the Landauer limit accounting for the fidelity of the output. For the reverse process, we note that the experimental $L$-state bit creation protocol has $\langle W\rangle>0$, and it is therefore beneficial to ignore the $L$-state inputs for the best aggregate performance. In this manner, we obtain $\langle W\rangle=-0.094 k_{\mathrm{B}} T$ for bit creation, which amounts to $30 \%$ of the entropy deficit in the input ensemble.

We note that the protocols targeting the $L$ state generally perform worse. This behavior is reproduced by the simulations (see values in Table I) and can therefore be primarily attributed to the strong asymmetry of the potential. It should be fairly straightforward to approach the theoretical ideal values with the present experimental hardware by slowing down the protocols and adjusting the barrier height to limit leakage-type errors. Similarly, the effect of the asymmetry of the potential can be compensated for in the design of the control waveforms [43].

In conclusion, we presented a trajectory-level analysis of the thermodynamics of information erasure in a superconducting flux logic device, where a double-well potential arises naturally through a combination of the Josephson effect and flux quantization. We calibrated a microscopic model of the device energetics and evaluated detailed work histograms for bit erasure protocols in a parameter regime where a metastable two-state approximation is valid throughout the protocol. We also demonstrated that a simple dynamical model, based on the calibrated potential and barrier activation, explains all experimental observations in detail. This sets the stage for designing and diagnosing efficient thermodynamic computing based on superconducting devices.

In the future, the study of speed-efficiency tradeoffs would appear to be a fruitful research direction with fundamental science and application-driven interest. Both classical [5] and quantum $[2,7,46]$ speed limits have been discussed in the literature. In this initial study, the execution speed of the bit reset was constrained by the limitations of the DC SQUID readout scheme. Future experiments employing either dispersive readout [47] with a wideband quantum-limited preamplifier [48] or thermal detectors [49] will enable gigahertz-scale clock rates while still maintaining fraction-of- $k_{\mathrm{B}} T$ excess dissipation and a similar resolution for the extracted thermodynamical quantities.

We thank A. Boyd and C. Jarzynski for helpful discussions. J.P.C. thanks the Santa Fe Institute and J.P.C., O.-P.S., M.H.M., R.K., W.F., G.W., and M.L.R. thank the Telluride Science Research Center for their hospitality during visits. This material is based upon work supported by, or in part by, the U.S. Army Research Laboratory and the U.S. Army Research Office under Contracts No. W911NF-13-1-0390 and No. W911NF-18-1-0028.
[1] M. P. Mills, The cloud begins with coal-Big data, big networks, big infrastructure, and big power: An overview of the electricity used by the global digital ecosystem, Digital Power Group report, 2013 (unpublished).

[2] S. Lloyd, Ultimate physical limits to computation, Nature (London) 406, 1047 (2000).

[3] R. Landauer, Irreversibility and heat generation in the computing process, IBM J. Res. Dev. 5, 183 (1961).

[4] A. Bérut, A. Arakelyan, A. Petrosyan, S. Ciliberto, R. Dillenschneider, and E. Lutz, Experimental verification of Landauer's principle linking information and thermodynamics, Nature (London) 483, 187 (2012).

[5] Y. Jun, M. Gavrilov, and J. Bechhoefer, High-Precision Test of Landauer's Principle in a Feedback Trap, Phys. Rev. Lett. 113, 190601 (2014).

[6] J. Hong, B. Lambson, S. Dhuey, and J. Bokor, Experimental test of Landauer's principle in single-bit operations on nanomagnetic memory bits, Sci. Adv. 2, e1501492 (2016).

[7] R. Gaudenzi, E. Burzurí, S. Maegawa, H. S. J. van der Zant, and F. Luis, Quantum Landauer erasure with a molecular nanomagnet, Nat. Phys. 14, 565 (2018).

[8] L. L. Yan, T. P. Xiong, K. Rehan, F. Zhou, D. F. Liang, L. Chen, J. Q. Zhang, W. L. Yang, Z. H. Ma, and M. Feng, Single-Atom Demonstration of the Quantum Landauer Principle, Phys. Rev. Lett. 120, 210601 (2018).

[9] A. Hofmann, V. F. Maisi, C. Rössler, J. Basset, T. Krähenmann, P. Märki, T. Ihn, K. Ensslin, C. Reichl, and W. Wegscheider, Equilibrium free energy measurement of a confined electron driven out of equilibrium, Phys. Rev. B 93, 035425 (2016).
[10] M. Gopalkrishnan, A cost/speed/reliability tradeoff to erasing, Entropy 18, 165 (2016).

[11] C. Jarzynski, Nonequilibrium Equality for Free Energy Differences, Phys. Rev. Lett. 78, 2690 (1997).

[12] G. E. Crooks, Entropy production fluctuation theorem and the nonequilibrium work relation for free energy differences, Phys. Rev. E 60, 2721 (1999).

[13] J. Liphardt, S. Dumont, S. B. Smith, I. Tinoco, and C. Bustamante, Equilibrium information from nonequilibrium measurements in an experimental test of Jarzynski's equality, Science 296, 1832 (2002).

[14] G. M. Wang, E. M. Sevick, E. Mittag, D. J. Searles, and D. J. Evans, Experimental Demonstration of Violations of the Second Law of Thermodynamics for Small Systems and Short Time Scales, Phys. Rev. Lett. 89, 050601 (2002).

[15] F. Douarche, S. Ciliberto, A. Petrosyan, and I. Rabbiosi, An experimental test of the Jarzynski equality in a mechanical experiment, Europhys. Lett. 70, 593 (2005).

[16] N. Garnier and S. Ciliberto, Nonequilibrium fluctuations in a resistor, Phys. Rev. E 71, 060101(R) (2005).

[17] V. Blickle, T. Speck, L. Helden, U. Seifert, and C. Bechinger, Thermodynamics of a Colloidal Particle in a TimeDependent Nonharmonic Potential, Phys. Rev. Lett. 96, 070603 (2006).

[18] O.-P. Saira, Y. Yoon, T. Tanttu, M. Möttönen, D. V. Averin, and J. P. Pekola, Test of the Jarzynski and Crooks Fluctuation Relations in an Electronic System, Phys. Rev. Lett. 109, 180601 (2012). 
[19] B. Küng, C. Rössler, M. Beck, M. Marthaler, D. S. Golubev, Y. Utsumi, T. Ihn, and K. Ensslin, Irreversibility on the Level of Single-Electron Tunneling, Phys. Rev. X 2, 011001 (2012).

[20] I. Chiorescu, Y. Nakamura, C. J. P. M. Harmans, and J. E. Mooij, Coherent quantum dynamics of a superconducting flux qubit, Science 299, 1869 (2003).

[21] N. Cottet, S. Jezouin, L. Bretheau, P. Campagne-Ibarcq, Q. Ficheux, J. Anders, A. Auffèves, R. Azouit, P. Rouchon, and B. Huard, Observing a quantum Maxwell demon at work, Proc. Natl. Acad. Sci. USA 114, 7561 (2017).

[22] Y. Masuyama, K. Funo, Y. Murashita, A. Noguchi, S. Kono, Y. Tabuchi, R. Yamazaki, M. Ueda, and Y. Nakamura, Information-to-work conversion by Maxwell's demon in a superconducting circuit quantum electrodynamical system, Nat. Commun. 9, 1291 (2018).

[23] A. D. King, J. Carrasquilla, J. Raymond, I. Ozfidan, E. Andriyash, A. Berkley, M. Reis, T. Lanting, R. Harris, F. Altomare et al., Observation of topological phenomena in a programmable lattice of 1,800 qubits, Nature (London) 560, 456 (2018).

[24] R. Harris, Y. Sato, A. J. Berkley, M. Reis, F. Altomare, M. H. Amin, K. Boothby, P. Bunyk, C. Deng, C. Enderud et al., Phase transitions in a programmable quantum spin glass simulator, Science 361, 162 (2018).

[25] C. J. Burroughs, P. D. Dresselhaus, A. Rufenacht, D. Olaya, M. M. Elsbury, Y. H. Tang, and S. P. Benz, NIST $10 \mathrm{~V}$ programmable Josephson voltage standard system, IEEE Trans. Instrum. Meas. 60, 2482 (2011).

[26] D. E. Kirichenko, S. Sarwana, and A. F. Kirichenko, Zero static power dissipation biasing of RSFQ circuits, IEEE Trans. Appl. Supercond. 21, 776 (2011).

[27] S. Boixo, T. F. Rønnow, S. V. Isakov, Z. Wang, D. Wecker, D. A. Lidar, J. M. Martinis, and M. Troyer, Evidence for quantum annealing with more than one hundred qubits, Nat. Phys. 10, 218 (2014).

[28] S. O. Valenzuela, W. D. Oliver, D. M. Berns, K. K. Berggren, L. S. Levitov, and T. P. Orlando, Microwave-induced cooling of a superconducting qubit, Science 314, 1589 (2006).

[29] J. P. Turneaure and I. Weissman, Microwave surface resistance of superconducting niobium, J. Appl. Phys. 39, 4417 (1968).

[30] A. Megrant, C. Neill, R. Barends, B. Chiaro, Y. Chen, L. Feigl, J. Kelly, E. Lucero, M. Mariantoni, P. J. J. O’Malley, D. Sank, A. Vainsencher, J. Wenner, T. C. White, Y. Yin, J. Zhao, C. J. Palmstrøm, J. M. Martinis, and A. N. Cleland, Planar superconducting resonators with internal quality factors above one million, Appl. Phys. Lett. 100, 113510 (2012).

[31] S. Han, J. Lapointe, and J. E. Lukens, Thermal Activation in a Two-Dimensional Potential, Phys. Rev. Lett. 63, 1712 (1989).

[32] S. Han, J. Lapointe, and J. E. Lukens, Effect of a twodimensional potential on the rate of thermally induced escape over the potential barrier, Phys. Rev. B 46, 6338 (1992).

[33] R. Harris, J. Johansson, A. J. Berkley, M. W. Johnson, T. Lanting, S. Han, P. Bunyk, E. Ladizinsky, T. Oh, I. Perminov, E. Tolkacheva, S. Uchaikin, E. M. Chapple, C. Enderud,
C. Rich, M. Thom, J. Wang, B. Wilson, and G. Rose, Experimental demonstration of a robust and scalable flux qubit, Phys. Rev. B 81, 134510 (2010).

[34] S.-X. Li, Y. Yu, Y. Zhang, W. Qiu, S. Han, and Z. Wang, Quantitative Study of Macroscopic Quantum Tunneling in a dc SQUID: A System with Two Degrees of Freedom, Phys. Rev. Lett. 89, 098301 (2002).

[35] D. Massarotti, L. Longobardi, L. Galletti, D. Stornaiuolo, D. Montemurro, G. Pepe, G. Rotoli, A. Barone, and F. Tafuri, Escape dynamics in moderately damped Josephson junctions (review article), Low Temp. Phys. 38, 263 (2012).

[36] A. B. Boyd and J. P. Crutchfield, Maxwell Demon Dynamics: Deterministic Chaos, the Szilard Map, and the Intelligence of Thermodynamic Systems, Phys. Rev. Lett. 116, 190601 (2016).

[37] A. B. Boyd, D. Mandal, and J. P. Crutchfield, Thermodynamics of Modularity: Structural Costs Beyond the Landauer Bound, Phys. Rev. X 8, 031036 (2018).

[38] I. Affleck, Quantum-Statistical Metastability, Phys. Rev. Lett. 46, 388 (1981).

[39] H. Grabert and U. Weiss, Crossover from Thermal Hopping to Quantum Tunneling, Phys. Rev. Lett. 53, 1787 (1984).

[40] P. Hanggi, H. Grabert, G.-L. Ingold, and U. Weiss, Quantum Theory of Activated Events in Presence of Long-Time Memory, Phys. Rev. Lett. 55, 761 (1985).

[41] R. Rouse, S. Han, and J. E. Lukens, Observation of Resonant Tunneling between Macroscopically Distinct Quantum Levels, Phys. Rev. Lett. 75, 1614 (1995).

[42] M. Neeley, M. Ansmann, R. C. Bialczak, M. Hofheinz, N. Katz, E. Lucero, A. O'Connell, H. Wang, A. N. Cleland, and J. M. Martinis, Transformed dissipation in superconducting quantum circuits, Phys. Rev. B 77, 180508(R) (2008).

[43] G. Wimsatt, O.-P. Saira, A. B. Boyd, M. H. Matheny, S. Han, M. L. Roukes, and J. P. Crutchfield, Harnessing fluctuations in thermodynamic computing via time-reversal symmetries, arXiv: 1906.11973.

[44] J. V. Koski, V. F. Maisi, J. P. Pekola, and D. V. Averin, Experimental realization of a Szilard engine with a single electron, Proc. Natl. Acad. Sci. USA 111, 13786 (2014).

[45] E. Ben-Jacob, D. J. Bergman, Y. Imry, B. J. Matkowsky, and Z. Schuss, Thermal activation from the fluxoid and the voltage states of dc SQUIDs, J. Appl. Phys. 54, 6533 (1983).

[46] C. Jarzynski, Generating shortcuts to adiabaticity in quantum and classical dynamics, Phys. Rev. A 88, 040101(R) (2013).

[47] C. M. Quintana, Y. Chen, D. Sank, A. G. Petukhov, T. C. White, D. Kafri, B. Chiaro, A. Megrant, R. Barends, B. Campbell et al., Observation of Classical-Quantum Crossover of $1 / f$ Flux Noise and Its Paramagnetic Temperature Dependence, Phys. Rev. Lett. 118, 057702 (2017).

[48] B. Ho Eom, P. K. Day, H. G. LeDuc, and J. Zmuidzinas, A wideband, low-noise superconducting amplifier with high dynamic range, Nat. Phys. 8, 623 (2012).

[49] J. P. Pekola, P. Solinas, A. Shnirman, and D. V. Averin, Calorimetric measurement of work in a quantum system, New J. Phys. 15, 115006 (2013). 